\title{
Microsurgical anatomy of the extracranial-extradural origin of the posterior inferior cerebellar artery
}

\author{
Andrew D. Fine, M.D., Alberto Cardoso, M.D., and Albert L. Rhoton, Jr., M.D. \\ Department of Neurological Surgery, University of Florida, Gainesville, Florida
}

Object. The authors describe the microsurgical anatomy of the posterior inferior cerebellar artery (PICA) with an extradural origin and discuss its importance as a common variation.

Methods. The microsurgical anatomy of paired PICAs with an extradural origin were examined.

Conclusions. Five to $20 \%$ of PICAs have an extradural origin. In the case described, both PICAs arose extradurally from the third segment of the vertebral artery (VA). Both origins were less than $1 \mathrm{~cm}$ proximal to the site at which the VA penetrated the dura and neither PICA gave rise to extradural branches. Extradurally the PICAs coursed parallel to the VA and the C-1 nerve and the three structures penetrated the dura together. Intradurally, the PICAs remained lateral and posterior to the brainstem, whereas, in the common PICA configuration, the first segment of the PICA courses anterior to the medulla. Neither PICA sent branches to the anterior brainstem, which is commonly found in PICAs with an intradural origin. There were no soft-tissue or bone anomalies.

Key Words * posterior inferior cerebellar artery * microsurgery * anatomical study * vertebral artery * variation

The location of the origin of the posterior inferior cerebellar artery (PICA) is highly variable. An extracranial-extradural origin from the vertebral artery (VA) is not rare. $[5,18,21,23,28]$ The anatomy of the PICA with an intradural origin has been well described, but a detailed description of the PICA that originates extradurally is lacking. The PICA with an extradural origin may be encountered during a lateral suboccipital, far-lateral, or upper cervical approach. Vertebral artery dissections tend to originate extradurally and may pose a greater risk to the PICA and of brainstem infarction when the PICA origin is extradural.[6,13] In this paper we examine the microsurgical anatomy of paired PICAs with an extradural origin. Authors of earlier reports have focused largely on the relationship between the origin of the PICA and the dura. In our report we also examine the relationship between the PICA with an extradural origin and the adjacent structures encountered in the operative approaches to the region and adjacent posterior meningeal and posterior spinal arteries. The effect of the extradural origin on the different segments of the PICA is also reviewed.

\section{MATERIALS AND METHODS}


One formalin-fixed adult head, in which bilateral extradural PICA origins were found, provided the material for examination. Blood clots were removed from the vessels by perfusion with water after which the arteries were injected with red latex and the veins with blue latex. Magnifications ranging from X 3 to $\mathrm{X} 20$ facilitated the dissection and measurements.

\section{RESULTS}

\section{Vertebral Artery Relationships}

The VA has four segments. The first segment originates as the first branch of the subclavian artery and courses upward and backward toward the C-6 transverse process. The second segment begins where the VA enters the transverse foramen of C- 6 and continues vertically to the transverse foramen of the axis. From there it runs laterally and upward to the transverse foramen of the atlas.[11] The third segment, the segment from which a PICA with an extradural origin usually arises, begins as the VA exits the transverse foramen of $\mathrm{C}-1$ on the medial side of the lateral rectus muscle of the head. This segment runs horizontally and medially above the posterior arch of $\mathrm{C}-1$ and passes beneath the posterior atlantooccipital membrane before turning upward and anteriorly to reach the site of dural penetration, where the dura thickens to form a fibrous dural ring around the artery.[7] The third segment is covered by the semispinal muscle of the head and courses within the depths of the suboccipital triangle formed by the borders of the greater posterior rectus muscle of the head and the superior and inferior oblique muscles (Fig. 1).[7,11,14] The fourth and final segment is located inside the dura within the subarachnoid space.

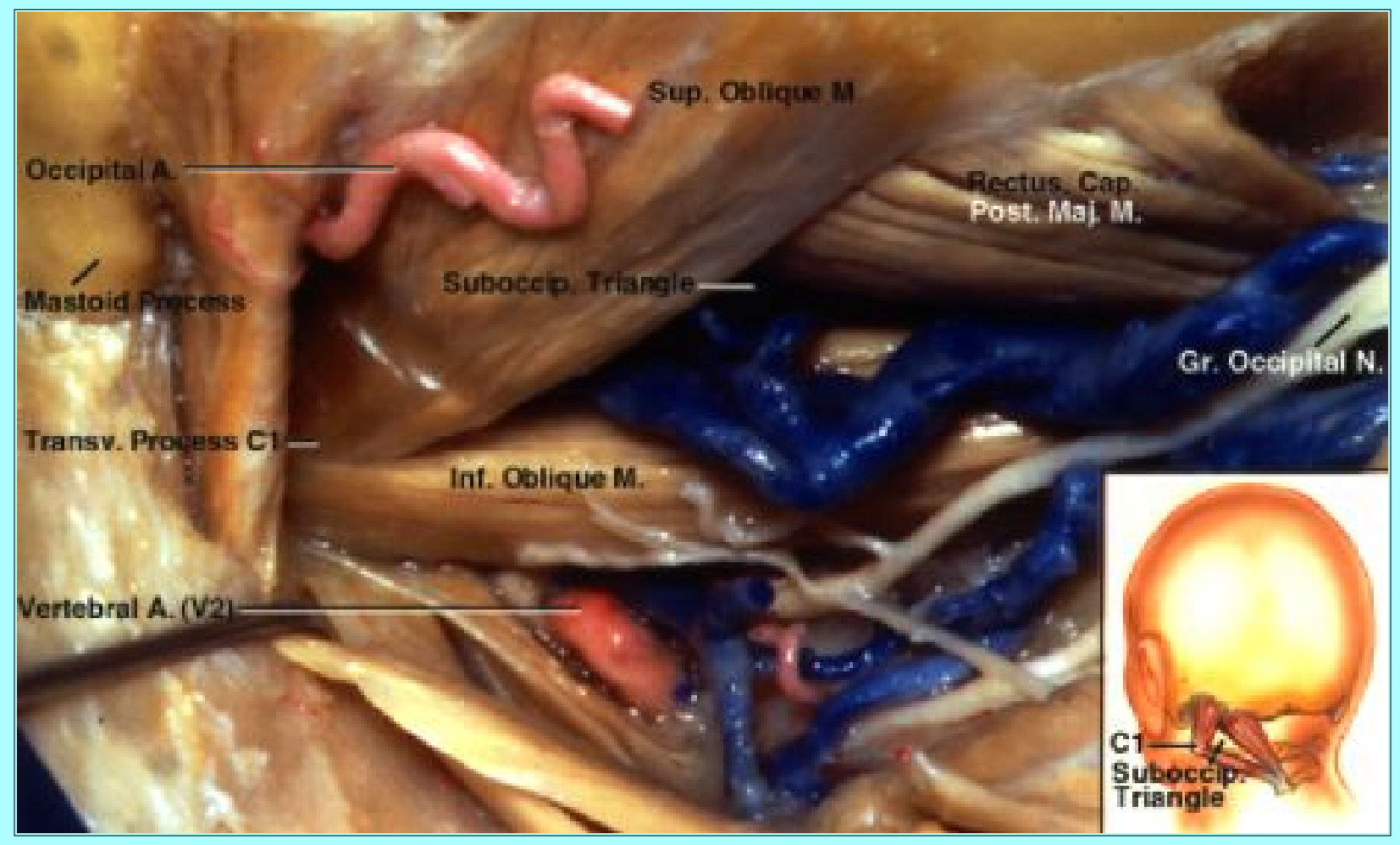

Fig. 1. Photograph showing that the terminal extradural segment of the VA, the segment from which a PICA with an extradural origin arises, is located in the depths of the suboccipital triangle. The left suboccipital triangle has been exposed by reflecting the sternocleidomastoid and splenius and longissimus capitis muscles. The triangle is bordered by three muscles: the inferior oblique below, the superior oblique above, and the greater posterior rectus muscle of the head (Rectus. Cap.) medially. The segment of the left VA 
within the triangle is covered by the cervical and vertebral venous plexus. The segment of the VA, ascending between $\mathrm{C}-1$ and $\mathrm{C}-2$, is exposed below the inferior oblique muscle and the greater occipital nerve. The VA is inclined laterally as it ascends between the C-2 and $\mathrm{C}-1$ transverse processes. $\mathrm{A}=$ artery; Gr. = greater; Inf. = inferior; $\mathrm{M}=$ muscle; Maj. = major; $\mathrm{N}=$ nerve; Post. $=$ posterior; Suboccip. $=$ suboccipital; Sup. $=$ superior; Transv. $=$ transverse.

\section{Origin and Extradural Course}

The PICA, defined as the cerebellar artery arising from the VA, was present bilaterally in this specimen (Fig. 2).[18] The PICAs arose from the VAs after the latter had crossed above the posterior arch of C-1 at the point at which the VAs turned upward and anteriorly to penetrate the dura.

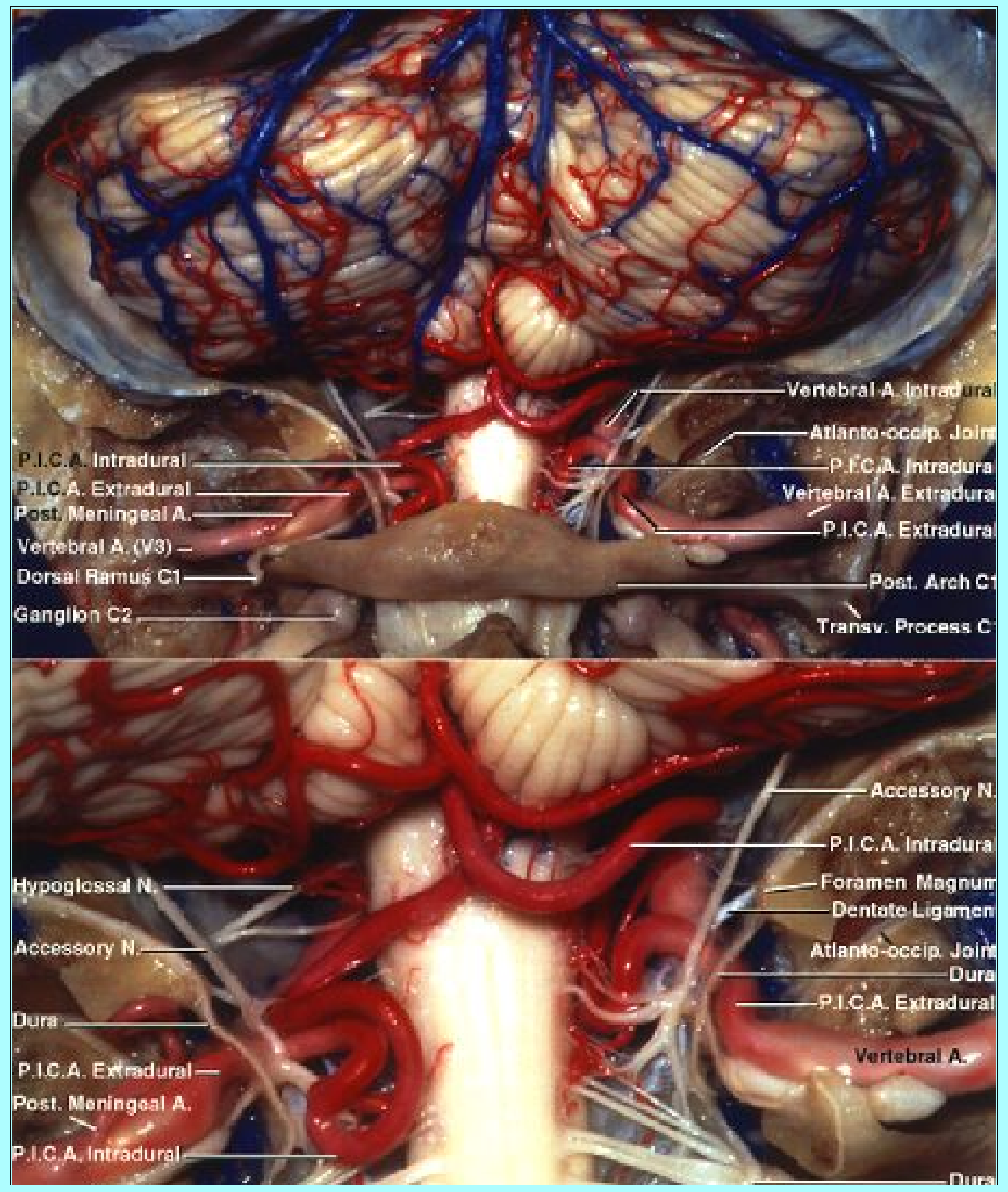




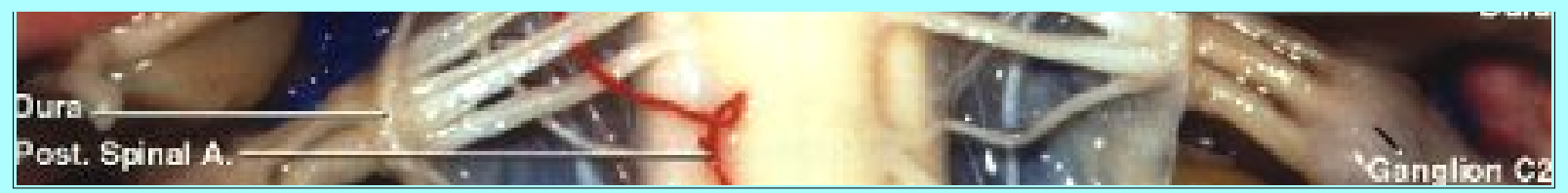

Fig. 2. Upper: Posterior view of the suboccipital surface of the cerebellum, which is supplied by the PICAs. A wide suboccipital craniotomy and C-1 posterior arch removal has been completed. Both PICAs arise in an extradural location and penetrate the dura along the posterior surface of the VAs. They course behind the medulla and around the cerebellar tonsil and ascend to supply the suboccipital surface of the cerebellum. Lower: Enlarged posterior view. Both PICAs arise from the vertical part of the third segment of the VAs and pursue a similar extradural course parallel and behind the VAs. Intradurally, the PICAs and VAs separate: the PICAs course medially to reach the posterior surface of the medulla and the VAs pass anterior to reach the ventral brainstem. $\mathrm{A}=$ artery; Atlanto-occip. $=$ atlantooccipital; $\mathrm{N}=$ nerve; Post. $=$ posterior; Transv. $=$ transverse.

The right PICA arose $7 \mathrm{~mm}$ proximal to the dural ring (Fig. 3 left). The VA at that point was $5.5 \mathrm{~mm}$ in diameter and the PICA diameter at the origin was $2.5 \mathrm{~mm}$. Its origin corresponded to a position $8 \mathrm{~mm}$ inferior to the foramen magnum. The left PICA arose $6 \mathrm{~mm}$ proximal to the dural ring (Fig. 3 right).

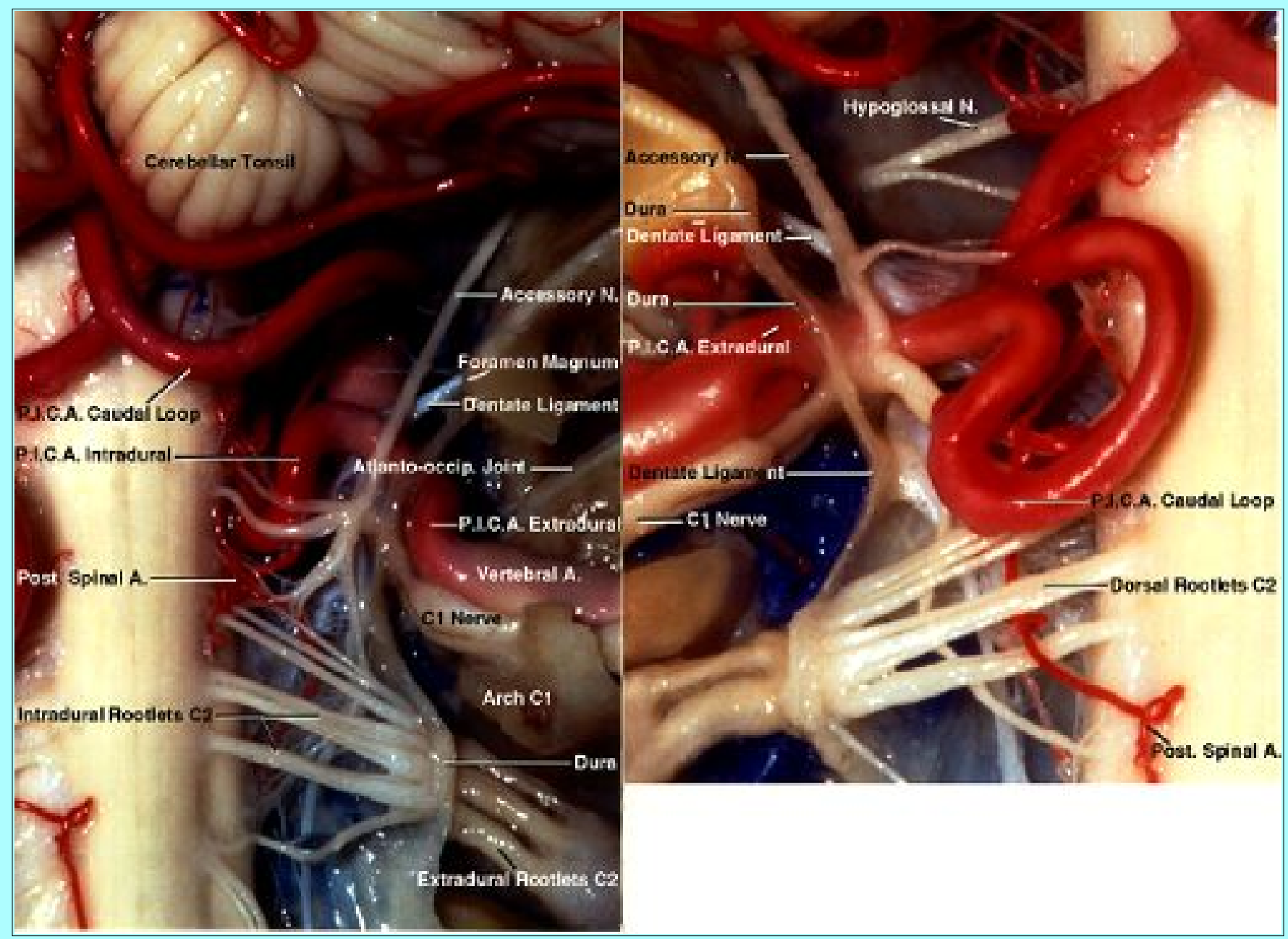

Fig. 3. Left: Enlarged view of the right PICA. The PICA penetrates the dura and passes in front of the spinal portion of the accessory nerve and behind the upper attachment of the dentate ligament. The VA penetrates the dura in front of both the accessory nerve and the dentate ligament. The C-1 nerve root passes below the extradural origin of the PICA. Right: 
The left PICA penetrates the dura medial to the atlantooccipital joint and has no extradural branches. The PICA shares the site of dural penetration with the VA and the C-1 nerve root. After penetrating the dura, the PICA loops caudally to the level of the C-2 dorsal root. $\mathrm{A}=$ artery; N. = nerve; Post. $=$ posterior.

At the PICA origin, the VA was $5 \mathrm{~mm}$ and the PICA was $2.2 \mathrm{~mm}$ in diameter. Its origin was $6 \mathrm{~mm}$ inferior to the foramen magnum. These measurements are within previously reported ranges.[18] Both PICAs arose from the posterior surface of the VAs, superior to the C-1 nerve roots and below the medial edge of the atlantooccipital joints (Figs. 2 and 4). The most common extradural PICA origin is on the lateral or posterior wall of the VA.[11,18]

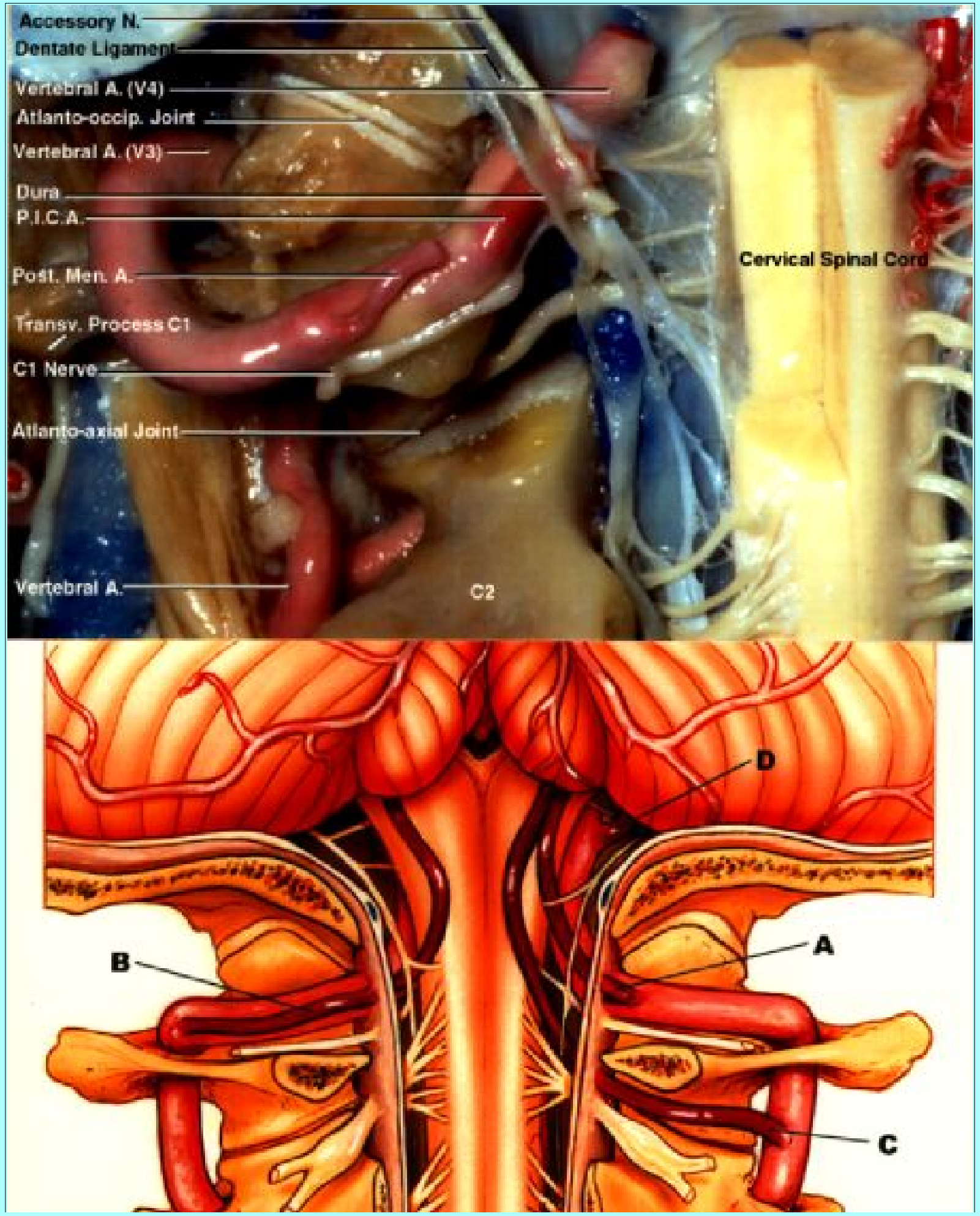




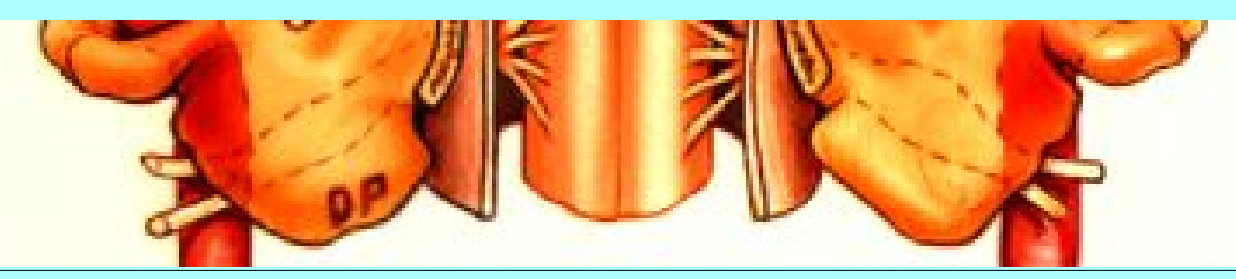

Fig. 4. Upper: The brainstem, the intradural segment of the VA and PICA, and the left dorsal quadrant of the cervical cord have been removed to show the relationship of the VA, dentate ligament, ventral rootlets of $\mathrm{C}-1$, and the accessory rootlets to the extradural and initial intradural segment of the PICAs. The VA passes in front of the accessory nerve and the upper attachment of the dentate ligament. The initial intradural segment of the PICA passes behind the dentate ligament and in front of the accessory nerve. The posterior meningeal artery arises below the atlantooccipital joint, after the VA has ascended through the transverse foramen of $\mathrm{C}-1 . \mathrm{A}=$ artery; atlanto-occip. = atlantooccipital; Post. Men. = posterior meningeal. Lower: Posterior view of the foramen magnum and VAs. A PICA with an extradural origin may arise just outside the dura (A), farther laterally above the transverse foramen of the atlas (B), or at the point at which the VA ascends between the transverse foramina of C-2 and C-1 (C). The typical origin is intradural (D).

Extradurally, the PICAs coursed parallel to the VAs with the PICAs and VAs remaining in contact prior to reaching the site of dural penetration. There were no extradural PICA branches on either side. The PICAs passed beneath the posterior atlantooccipital membrane with the VAs before entering the dura. On the left side, the posterior meningeal artery originated from the VA $15 \mathrm{~mm}$ proximal to the dural ring and was $0.8 \mathrm{~mm}$ in diameter (Fig. 2 lower). On the right side, the posterior meningeal artery was not observed to arise from either the PICA or the VA, making it likely that the occipital artery supplied the dura in this area.[28] Muscular branches ranging from 0.2 to $0.4 \mathrm{~mm}$ in diameter arose from the horizontal portion of $\mathrm{V}_{3}$. No muscular branches arose from the extradural portion of the PICA.

\section{Intradural Course}

The PICAs, VAs, and C-1 nerve roots passed through the dura together on both sides, with the C-1 nerve being the most dorsal and caudal. The PICAs pierced the dura on the posterior aspect of the VAs, above and anterior to the $\mathrm{C}-1$ nerves (Figs. 2 lower, 3 , and 4 upper). The accessory nerve passed dorsally and the dentate ligament ventrally with respect to the initial intradural segment of the PICA, and the dentate ligament separated the initial intradural segment of the VA and PICA on both sides (Figs. 3 and 4 upper).

Once intradural, the right and left PICAs became less symmetrical. The initial intradural portion of the PICAs had two branches on the right side and three on the left, all terminating on the lateral and posterior aspect of the upper spinal cord and medulla oblongata (Figs. 2 lower and 3). The first of these branches was the posterior spinal artery, which arose below the level of the foramen magnum on each side and passed to the posterolateral surface of the spinal cord, where it gave rise to ascending and descending branches that coursed along the dorsal root entry zone. The largest of these branches was on the left, had a caliber of $0.5 \mathrm{~mm}$ at its origin, and terminated along the medial edge of the dorsal roots (Figs. 2 lower and 3 right). The anterior medulla oblongata derived the majority of its arterial supply from the VAs without a contribution from the PICAs. Both PICAs arose caudal and coursed dorsal to the inferior olive, rather than following the more typical pattern seen in PICAs having an intradural origin, in which the PICAs arise at the level of, and course beside, the inferior olive. Both arteries entered the dura at a level that corresponded to the lateral medullary segment of the PICA. Neither PICA had an anterior medullary 
segment.[18] Both PICAs reached the posterior surface of the medulla oblongata by passing between the rootlets of the spinal accessory nerve (Figs. 2 lower, 3, 4 upper, and 5).

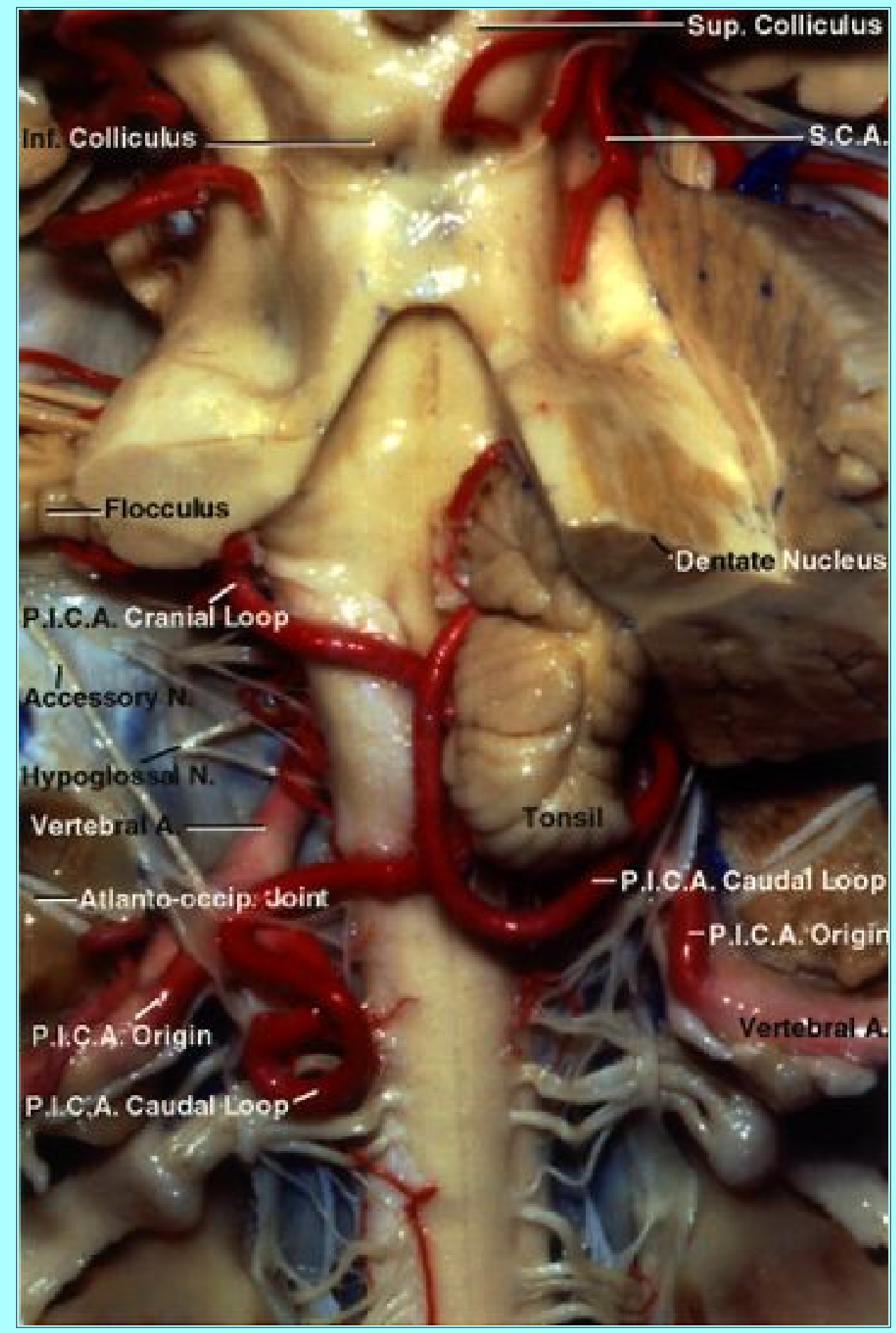

Fig. 5. Posterior view showing the relationships between the PICAs and the lower pole of the tonsils and the fourth ventricle. On the right, the caudal loop approximates the level of the lower pole of the tonsil, but on the left the caudal loop is located well below the lower pole and the foramen magnum. A = artery; Atlanto-occip. = atlantooccipital; Inf. = inferior; $\mathrm{N}=$ nerve; S.C.A. = superior cerebellar artery; Sup. = superior.

The lateral medullary segment of the PICA ended and the tonsillomedullary segment began as the arteries passed dorsal to the rootlets of the accessory nerve. The tonsillomedullary segment coursed around the lower pole of the tonsil, turned upward along the anteromedial aspect of the tonsil, and ended 
midway between the upper and lower poles of the tonsil and behind the inferior half of the roof of the fourth ventricle. $[9,20,21]$ The right tonsillomedullary segment was the site of a caudal loop[18] that dipped to the level of the foramen magnum $7 \mathrm{~mm}$ below the lower pole of the tonsil. The left PICA pursued a complex tortuous course, descending to form a caudal loop behind the left C-2 dorsal root, 17 $\mathrm{mm}$ below the foramen magnum, before passing upward and crossing in front of the left tonsil to reach the area behind the roof of the fourth ventricle (Figs. 2 lower and 3 right). Contrary to prior reports, the caudal loop is of little predictive value in assessing the position of the cerebellar tonsils and their relationship to the foramen magnum. $[9,18,21]$ On the right side, the caudal loop was located directly below the cerebellar tonsil; however, on the left side it was located behind the C-2 dorsal root lateral to the area below the left tonsil. In a series of 42 PICAs examined by Lister, et al.,[18] the caudal limit of this segment was located at the caudal pole of the tonsil in 11 PICAs, inferior to the caudal pole in eight, and superior to the caudal pole in 23 PICAs.

The next segment, the telovelotonsilar segment, began at the midlevel of the cerebellar tonsil and behind the inferior half of the roof of the fourth ventricle. This segment ended where the PICA exited the telovelotonsillar fissure located at the point at which the tela choroidea and inferior medullary velum, which form the lower half of the roof of the fourth ventricle, wrap around the upper pole of the tonsil. This segment formed a rostrally convex cranial loop around the upper pole of the tonsil on both sides. The ascending proximal limb of this loop ascended to the level of the fastigium of the fourth ventricle on both sides before turning downward. The distal descending limb proceeded posteriorly in the telovelotonsilar fissure (Fig. 5). Both PICAs gave rise to branches to the tela choroidea and the choroid plexus as expected.[10] The cortical segment of the PICAs began at their exit from the telovelotonsilar fissure, where they gave rise to medial and lateral trunks that supplied the vermis and hemispheres.

\section{DISCUSSION}

The PICA has a highly variable origin. Authors of the two prior studies of PICA origin did not give a detailed account of an extradural origin.[18,21] The relative frequency of an extradural origin has led to its incidental identification in association with other conditions such as aneurysms; however, such reports have not dealt with anatomical considerations that are important to surgery. When defined as the cerebellar artery arising from the VA, the absence of PICAs is reported in 2 to $26 \%$ of cases.[10,18] The PICA's most common point of origin is approximately 16 or $17 \mathrm{~mm}$ proximal to the vertebrobasilar junction, but many variations exist (Fig. 4 lower).[18] Duplicate PICAs, a VA termination at the PICA, extradural origins at the $\mathrm{C}-1$ and $\mathrm{C}-2$ levels, origins at the hypoglossal, proatlantal, or posterior meningeal arteries, and at all points along the intradural VA are among the variations reported.[1,17,19,21,22,28] Bilateral extradural origins at the C-1 level, as reported here, also were described in one other report.[17] Padget[24] and Lasjaunias and colleagues[16,17] reviewed the embryology of the many reported variations.

Knowing the configuration of an extradural origin for the PICA is important for several reasons. The occurrence rate of 5 to $20 \%$ in all PICAs make it a common variation, exposing this vessel to injury during posterior approaches to the upper cervical spine and lower brainstem and during exposure of the VA.[21,23] Although extradural PICAs do not send perforating branches to the anterior surface of the medulla oblongata, they still make an important contribution to the brainstem by supplying the lateral and posterior medulla. Aneurysms of the PICA origin may be missed with a single contralateral VA angiogram if the contrast agent does not reflux to fill a proximal PICA origin.[15,28] 
The exposure of the region has been described by many investigators.[2,3,5,12,14,15,26,27] Safely exposing this variant requires a knowledge of the relationship between the extradural PICA origin and the atlantooccipital joint, suboccipital triangle, and transverse process of $\mathrm{C}-1$. The muscular, posterior meningeal, and posterior spinal branches of the $\mathrm{V}_{3}$ segment should not be mistaken for an extradural PICA. $[9,23,25]$ Careful examination of angiographic studies of the PICA's course in relation to bone landmarks will aid in identifying an extradural origin. An extradural PICA origin should be suspected when the artery arises below the foramen magnum; however, this is not always indicative of an extradural origin because PICA origins located immediately inside the dura may also be seen below the level of the foramen magnum. Those PICAs arising outside the dura most commonly penetrate the dura between the atlas and the foramen magnum; however, there are several reports of extradural PICA origins at C-2 with the artery penetrating the dura between C-1 and C-2 (Fig. 4 lower).[5,27]

Vertebral artery dissection, once believed to be rare but now more commonly recognized, most commonly begins on the extradural $V_{3}$ segment from which a PICA may arise.[7,13,24,29] Often the dissection will progress to the dural ring and then stop, which and may lead to a Wallenberg or lateral medullary syndrome.[8] Progression beyond the dural ring places the patient at risk for subarachnoid hemorrhage.

\section{References}

1. Ahuja A, Graves VB, Crosby DL, et al: Anomalous origin of the posterior inferior cerebellar artery from the internal carotid artery. AJNR 13:1625-1626, 1992

2. Anson JA, Spetzler RF: Endarterectomy of the intradural vertebral artery via the far lateral approach. Neurosurgery 33:804-811, 1993

3. Baldwin HZ, Miller GC, van Loveren HR, et al: The far lateral/combined supra- and infratentorial approach. A human cadaveric prosection model for routes of access to the petroclival region and ventral brain stem. J Neurosurg 81:60-68, 1994

4. Ban M, Ueta H, Nakagawa Y, et al: A case of basilar artery occlusion associated with unilateral low origin of the posterior inferior cerebellar artery. Surg Neurol 26:501-504, 1986

5. Bertalanffy H, Seeger W: The dorsolateral, suboccipital, transcondylar approach to the lower clivus and anterior portion of the craniocervical junction. Neurosurgery 29:815-821, 1991

6. DeBehnke DJ, Brady W: Vertebral artery dissection due to minor neck trauma. J Emerg Med 12:27-31, 1994

7. de Oliveira E, Rhoton AL Jr, Peace D: Microsurgical anatomy of the region of the foramen magnum. Surg Neurol 24:293-352, 1985

8. Fisher CM, Karnes WE, Kubik CS: Lateral medullary infarction--the pattern of vascular occlusion. J Neuropathol Exp Neurol 20:323-379, 1961

9. Friedman DP: Abnormalities of the posterior inferior cerebellar artery: MR imaging findings. AJR 160:1257-1263, 1993

10. Fujii K, Lenkey C, Rhoton AL Jr: Microsurgical anatomy of the choroidal arteries. Fourth ventricle 
and cerebellopontine angles. J Neurosurg 52:504-524, 1980

11. Gabella G (ed): Gray's Anatomy, ed 38. New York: Churchill Livingstone, 1995, pp 1530-1534

12. George B, Dematons C, Cophignon J: Lateral approach to the anterior portion of the foramen magnum. Application to surgical removal of 14 benign tumors: technical note. Surg Neurol 29:484-490, 1988

13. Golueke P, Sclafani S, Phillips T, et al: Vertebral artery injury--diagnosis and management. J Trauma 27:856-865, 1987

14. Heary RF, Albert TJ, Ludwig SC, et al: Surgical anatomy of the vertebral arteries. Spine 21:2074-2080, 1996

15. Heros RC: Lateral suboccipital approach for vertebral and vertebrobasilar artery lesions. J Neurosurg 64:559-562, 1986

16. Lasjaunias P, Guibert-Trainer F, Braun JP: The pharyngo-cerebellar artery or ascending pharyngeal artery origin of the posterior inferior cerebellar artery. J Neuroradiol 8:317-325, 1981

17. Lasjaunias P, Vallee B, Person H, et al: The lateral spinal artery of the upper cervical spinal cord. Anatomy, normal variations, and angiographic aspects. J Neurosurg 63:235-241, 1985

18. Lister JR, Rhoton AL Jr, Matsushima T, et al: Microsurgical anatomy of the posterior inferior cerebellar artery. Neurosurgery 10:170-199, 1982

19. Manabe $\mathrm{H}$, Oda N, Ishii M, et al: The posterior inferior cerebellar artery originating from the internal carotid artery, associated with multiple aneurysms. Neuroradiology 33:513-515, 1991

20. Margolis MT, Newton TH: An angiographic sign of cerebellar tonsillar herniation. Neuroradiology 2:3-8, 1971

21. Margolis MT, Newton TH: The posterior inferior cerebellar artery, in Newton TH, Potts DG (eds): Radiology of the Skull and Brain. St Louis: Mosby, 1974, Vol 2, Bk 2, pp 1719-1775

22. Ogawa T, Fujita H, Inugami A, et al: Anomalous origin of the posterior inferior cerebellar artery from the posterior meningeal artery. AJNR 12:186, 1991

23. Osborn AG: Introduction to Cerebral Angiography. Philadelphia: Harper \& Row, 1980, pp $379-427$

24. Padget DH: The development of the cranial arteries in the human embryo. Contrib Embryol 32:207-262, 1948

25. Quint DJ, Spickler EM: Magnetic resonance demonstration of vertebral artery dissection. Report of two cases. J Neurosurg 72:964-967, 1990

26. Sen CN, Sekhar LN: An extreme lateral approach to intradural lesions of the cervical spine and foramen magnum. Neurosurgery 27:197-204, 1990

27. Spetzler RF, Grahm TW: Far lateral approach to the inferior clivus and the upper cervical region: technical note. BNI Q 6:35-38, 1990 
28. Tanaka A, Kimura M, Yoshinaga S, et al: Extracranial aneurysm of the posterior inferior cerebellar artery: case report. Neurosurgery 33:742-745, 1993

29. Wilkinson IMS: The vertebral artery. Extracranial and intracranial structure. Arch Neurol 27:392-396, 1972

Manuscript received February 2, 1999.

Accepted in final form May 19, 1999.

Address reprint requests to: Albert L. Rhoton, Jr., M.D., Department of Neurological Surgery, University of Florida College of Medicine, P.O. Box 100265, 100 South Newell Drive, L2-100, Gainesville, Florida 32610-0265. email: rhoton@ neurosurgery.ufl.edu. 